\title{
Fault Reconstruction Based on Sliding Mode Observer for Current Sensors of PMSM
}

\author{
Changfan Zhang, Huijun Liao, Xiangfei Li, Jian Sun, and Jing He \\ College of Electrical and Information Engineering, Hunan University of Technology, Zhuzhou, Hunan 412007, China
}

Correspondence should be addressed to Jing He; hejing@263.net

Received 6 January 2015; Revised 20 April 2015; Accepted 20 April 2015

Academic Editor: Mehmet Karakose

Copyright (C) 2016 Changfan Zhang et al. This is an open access article distributed under the Creative Commons Attribution License, which permits unrestricted use, distribution, and reproduction in any medium, provided the original work is properly cited.

\begin{abstract}
This paper deals with a method of phase current sensor fault reconstruction for permanent magnet synchronous motor (PMSM) drives. A new state variable is introduced so that an augmented system can be constructed to treat PMSM sensor faults as actuator faults. This method uses the PMSM two-phase stationary reference frame fault model and a sliding mode variable structure observer to reconstruct fault signals. A logic algorithm is built to isolate and identify the faulty sensor for a stator phase current fault after reconstructing the two-phase stationary reference frame fault signals, which allows the phase fault signals to be reconstructed. Simulation results are presented to illustrate the functionality of the theoretical developments.
\end{abstract}

\section{Introduction}

Permanent magnet synchronous motors (PMSMs) are an important category of electric machines, in which the rotor magnetization is created by permanent magnets attached to the rotor. Due to their high efficiency, high ratio of torque to weight, high power factor, faster response, and rugged construction, PMSMs are widely used for high performance variable speed motors in many industry applications. With the development of permanent magnet materials, especially the neodymium-iron-boron (Nd-Fe-B), which has high magnetic energy, high coercive force, and low price, the applications of PMSMs have become more extensive in recent years.

High performance systems usually have demanding requirements on availability, reliability, and survivability [1]. The reliable operation of PMSM is a primary concern for the entire ship system. Early detection of abnormalities in the PMSM drives will help to avoid failures. Indeed, detection, location, and analysis of faults play a very important role in reliable operation of electrical machines and are essential for major concerns such as efficiency and performance of applications involving PMSM drives. Sensor failure is one of several faults occurring in drive systems. Sensors are of great importance in the installation of monitoring and control systems. However, in most publications, detection of inverter faults or physical damage of the electrical machine is considered, rather than sensor faults. This paper proposes a method of phase current sensor fault detection and reconstruction for PMSM control systems.

In recent years, fault-tolerant control has been developed, which can be realized by reconstructing phase currents to substitute current feedback after the fault occurrence. For example, Lu et al. [2] reconstructed three-phase current based on DC bus sensors for fault-tolerant control for electric machines. Salmasi and Najafabadi [3] observed faulty phase stator currents based on an adaptive observer when there is only one normal phase current sensor. However, these designs do not separate fault signals from faulty sensors. Therefore, no further information can be obtained on the sensor state itself.

Due to the simplicity of the two-axis $d$-q-model, it has become the most widely used model in electric machine engineering controller design. The $d$-q-model offers significant convenience for control system design by transforming stationary symmetrical AC variables to DC variables in a rotating reference frame. Therefore, for electric machine sensor fault detection, the $d-q$ axis currents, $i_{d}$ and $i_{q}$, are assumed to be measured directly through sensors. Najafabadi et al. [4] detected $d-q$ axis current sensor faults based on an adaptive observer. Liu [5] used a nonlinear parity relation method for detection of additive faults for virtual sensors for $d-q$ 
axis currents. Huang [6], an unknown input observer was designed for detection of $q$ axis stator current residuals for induction motor. However, the two currents, $i_{d}$ and $i_{q}$, are not practically measurable. These two virtual sensing signals are calculated from the measured phase current, $i_{a b c}$, by applying a linear Clarke transformation. Abnormal changes in $i_{d}$ and $i_{q}$ may indicate a fault appearing in the phase current sensors, but this design will not provide more specific information. Furthermore, as the calculations of $i_{d}$ and $i_{q}$ are coupled, if a fault occurred in the measurement devices for $i_{a b c}$, consequential faults in $i_{d}$ and $i_{q}$ will appear simultaneously. Hence, it is relatively difficult to detect the abnormal signals in the measurement devices for $i_{a b c}$.

Sliding mode techniques are known for their robustness and insensitivity to the so-called matched uncertainty. Therefore these techniques offer great potential for robust fault detection and isolation (FDI) [7-10]. Faults are classified according to their physical locations into system, actuator, and sensor faults. Compared with actuators, sensors are passive elements in the sense that they only provide operational information about the system and do not affect the system behavior directly. Thus they have been less studied compared to actuator fault detection and isolation. This paper presents a method in which, using a newly designed filter, the sensor faults can be modeled as pseudoactuator faults. Then, using the transformed system structure and characteristics of the designed filter, a sliding mode observer is proposed to reconstruct the sensor fault in the system [11-13].

The paper is organized as follows. Section 2 describes a surface-mounted PMSM with current sensor faults. Section 3 proposes the method for reconstruction of faults. Section 4 presents simulation results and conclusions in Section 5 follow.

\section{System Formulation}

In the $\alpha-\beta$ rotor reference frame, a surface-mounted PMSM with current sensors faults can be expressed as the following dynamic model:

$$
\begin{aligned}
& \dot{x}(t)=A x(t)+B u(t)+E \psi, \\
& y(t)=C x(t)+N f_{s}(x, u, t),
\end{aligned}
$$

where $x(t), u(t), y(t), \psi$, and $f_{s}(x, u, t)$ are the state variables, inputs, outputs, rotor flux, and sensor faults, respectively. Then the matrices are defined as

$$
\begin{aligned}
& A=\left[\begin{array}{cc}
-\frac{R_{s}}{L_{\alpha}} & 0 \\
0 & -\frac{R_{s}}{L_{\beta}}
\end{array}\right], \\
& B=\left[\begin{array}{cc}
\frac{1}{L_{\alpha}} & 0 \\
0 & \frac{1}{L_{\beta}}
\end{array}\right],
\end{aligned}
$$

$$
\begin{aligned}
C & =\left[\begin{array}{ll}
1 & 0 \\
0 & 1
\end{array}\right], \\
E & =\left[\begin{array}{cc}
-\frac{\omega}{L_{\alpha}} & 0 \\
0 & -\frac{\omega}{L_{\beta}}
\end{array}\right], \\
N & =\left[\begin{array}{ll}
1 & 0 \\
0 & 1
\end{array}\right], \\
x & =\left[\begin{array}{l}
i_{\alpha} \\
i_{\beta}
\end{array}\right], \\
u & =\left[\begin{array}{l}
u_{\alpha} \\
u_{\beta}
\end{array}\right], \\
\psi & =\left[\begin{array}{l}
\psi_{\alpha} \\
\psi_{\beta}
\end{array}\right]=\left[\begin{array}{l}
-\psi_{f} \sin \theta_{r} \\
\psi_{f} \cos \theta_{r}
\end{array}\right], \\
y & =\left[\begin{array}{l}
i_{\alpha} \\
i_{\beta}
\end{array}\right], \\
f_{s} & =\left[\begin{array}{l}
f_{s \alpha} \\
f_{s \beta}
\end{array}\right]
\end{aligned}
$$

with

$$
\begin{aligned}
& L_{\alpha}=L_{0}+L_{1} \cos \left(2 \theta_{r}\right), \\
& L_{\beta}=L_{0}-L_{1} \cos \left(2 \theta_{r}\right), \\
& L_{0}=\frac{L_{d}+L_{q}}{2}, \\
& L_{1}=\frac{L_{d}-L_{q}}{2},
\end{aligned}
$$

where $i_{\alpha}, i_{\beta}, u_{\alpha}$, and $u_{\beta}$ are the $\alpha$ - $\beta$ axis currents and voltages, respectively; $\psi_{f}$ is the rotor permanent magnet flux linkage; $\theta_{r}$ is the electrical rotor angular position; $\psi_{\alpha}, \psi_{\beta}, f_{s \alpha}$, and $f_{s \beta}$ are the $\alpha$ - $\beta$ axis stator flux linkages and current sensor faults, respectively; $R_{s}$ is the stator resistance; $L_{\alpha}, L_{\beta}$, and $\omega$ are the $\alpha-\beta$ axis stator inductances and electrical rotor speed, respectively; and $L_{d}$ and $L_{q}$ are the stator $d$ and $q$ axis inductances, respectively. The surface-mounted PMSM stator winding shows the same electrical inductance on both $d$ and $q$ axes; that is, $L_{d}=L_{q}=L_{s}$, where $L_{s}$ is the stator inductance.

\section{Sensor Faults Reconstruction Based on Sliding Mode Variable Structure}

The section proposes a novel method to reconstruct the phase current sensor faults for PMSM drives. Before dealing with the faults, an extended $\alpha$ - $\beta$ axis fault model of PMSM is reformulated by using a transformation filter, which increases the system's state. Then, a sliding mode observer is constructed to 
reconstruct $\alpha-\beta$ fault signals. A logic algorithm is built to convert the reconstructed $\alpha-\beta$ fault signal into $a b c$-phase, so as to reconstruct phase current sensor faults.

3.1. Extended PMSM $\alpha-\beta$ Axis Fault Model and Sensor Faults Reconstruction. For system (1), a low-pass linear filter is introduced [11]:

$$
\dot{z}(t)=A_{s} z(t)+B_{s} y
$$

where $z$ is the new vector state; $A_{s}$ and $B_{s}$ are constant matrices, which are design parameters to be defined later; and $y$ is the output.

Combining systems (1) and (4), we have

$$
\dot{z}(t)=A_{s} z(t)+B_{s} C x(t)+B_{s} N f_{s}(x, u, t) .
$$

Then, the following augmented system can be obtained:

$$
\begin{aligned}
{\left[\begin{array}{c}
\dot{x} \\
\dot{z}
\end{array}\right] } & =\left[\begin{array}{cc}
A & 0 \\
B_{s} C & A_{s}
\end{array}\right]\left[\begin{array}{l}
x \\
z
\end{array}\right]+\left[\begin{array}{l}
B \\
0
\end{array}\right] u+\left[\begin{array}{c}
0 \\
B_{s} N
\end{array}\right] f_{s}+\left[\begin{array}{l}
E \\
0
\end{array}\right] \psi, \\
z & =\left[\begin{array}{ll}
0 & I
\end{array}\right]\left[\begin{array}{l}
x \\
z
\end{array}\right],
\end{aligned}
$$

where $f_{s}$ is the sensor faults of system (1). The new state variables and matrices in a compatible way with system (6) are as follows:

$$
\begin{aligned}
\bar{A} & =\left[\begin{array}{cc}
A & 0 \\
B_{s} C & A_{s}
\end{array}\right], \\
\bar{B} & =\left[\begin{array}{l}
B \\
0
\end{array}\right], \\
M & =\left[\begin{array}{c}
0 \\
B_{s} N
\end{array}\right], \\
\bar{E} & =\left[\begin{array}{l}
E \\
0
\end{array}\right], \\
\bar{C} & =\left[\begin{array}{ll}
0 & I
\end{array}\right], \\
\bar{x}(t) & =\left[\begin{array}{l}
x(t) \\
z(t)
\end{array}\right], \\
\bar{y}(t) & =z .
\end{aligned}
$$

Therefore, following this transformation, the system is extended and the initial sensor fault problem has become an actuator fault problem. The corresponding extended fault model is

$$
\begin{aligned}
& \dot{\bar{x}}(t)=\bar{A} \bar{x}(t)+\bar{B} u(t)+M f_{s}+\bar{E} \psi, \\
& \bar{y}(t)=\bar{C} \bar{x}(t) .
\end{aligned}
$$

This paper focuses only on sensor faults, which have been transformed into pseudoactuator faults in system (8).
Assumption $1\left((A, C)\right.$ is observable). When $B_{s}$ is a nonsingular matrix, if $(A, C)$ in system $(1)$ is observable, $(\bar{A}, \bar{C})$ in system (8) will still be observable [14].

Therefore, there is a matrix $L$, which makes $A_{0}=\bar{A}-L \bar{C}$ a stable matrix and satisfies the Lyapunov function:

$$
A_{0}^{T} P+P A_{0}=-Q
$$

where matrices $P, Q$ are symmetric positive definite.

Assumption 2. There is a matrix $F$, such that $P M=\bar{C}^{T} F^{T}$.

Assumption 3. A fault in the system is a bounded function, such that $\left\|f_{s}\right\| \leq \rho_{1}$, where $\rho_{1}$ is a selected scalar larger than 0 .

The sliding mode observer for system (8) can be designed as

$$
\begin{aligned}
\dot{\overline{\bar{x}}}(t)= & \bar{A} \widehat{\bar{x}}(t)+\bar{B} u(t)+L(\bar{y}(t)-\bar{C} \widehat{\bar{x}}(t))+M v \\
& +\bar{E} \psi, \\
\hat{\bar{y}}(t)= & \bar{C} \widehat{\bar{x}}(t),
\end{aligned}
$$

where $\hat{\bar{x}}(t)$ and $\hat{\bar{y}}(t)$ are the estimated states and outputs, respectively, and $v$ is the sliding mode signal, defined as

$$
v= \begin{cases}-\rho \frac{F e_{y}(t)}{\left\|F e_{y}(t)\right\|} & \text { if } e_{y} \neq 0 \\ 0 & \text { if } e_{y}=0,\end{cases}
$$

where $F$ is the observer gain and $\rho$ is a positive scalar. Both of these need to be designed.

If the state estimation errors are defined as $e(t)=\widehat{\bar{x}}(t)-$ $\bar{x}(t)$ and $e_{y}(t)=\bar{C} \hat{\bar{x}}(t)-\bar{y}(t)=\bar{C} e(t)$, then from (8) and (10), the state estimation error dynamical system is

$$
\dot{e}(t)=A_{0} e(t)+M\left(v-f_{s}\right) .
$$

The convergence of the above observer is guaranteed by the following proposition.

Proposition 4. Considering the system described by (8) and its observers described by (10), if Assumptions 1-3 hold, the parameter of the observer is selected according to the following criteria:

$$
\rho>\rho_{1}
$$

and thus $e$ is asymptotically convergent; that is, $\lim _{t \rightarrow \infty} e(t)=$ 0 .

Proof. For system (12), consider a Lyapunov function candidate $V_{1}=e^{T} P e$. The time derivative of $V_{1}$ along the trajectories of system (12) is

$$
\dot{V}_{1}=e^{T}\left(P A_{0}+A_{0} P\right) e+2 e^{T} P M v-2 e^{T} P M f_{s}
$$


and it follows that

$$
\begin{aligned}
& \dot{V}_{1} \leq-e^{T} Q e+2(F \bar{C} e)^{T}\left(v+f_{s}\right), \\
& \dot{V}_{1} \leq-e^{T} Q e-2\left(F e_{y}\right)^{T} \rho \frac{F e_{y}}{\left\|F e_{y}\right\|}+2\left\|F e_{y}\right\|\left\|f_{s}\right\|, \\
& \dot{V}_{1} \leq-e^{T} Q e-2\left\|F e_{y}\right\|\left(\rho-\left\|f_{s}\right\|\right) .
\end{aligned}
$$

Then, under Assumption 3, the following can be obtained from (15):

$$
\dot{V}_{1} \leq-e^{T} Q e-2\left\|F e_{y}\right\|\left(\rho-\rho_{1}\right) \leq-e^{T} Q e,
$$

from which $\dot{V}_{1}$ is negative definite. From the Lyapunov theorem, observer (10) is designed asymptotically stable.

It can be known from above that $e(t)$ will make asymptotic convergence to zero; that is, $\lim _{t \rightarrow \infty} e(t)=0$.

This completes the proof.

Proposition 4 implies that $e$ is bounded; that is, $t_{0}$ exists, and when $t>t_{0}$,

$$
\|e\| \leq \delta_{1}
$$

where $\delta_{1}$ is a finite positive scalar.

Remark 5. The obtained control algorithm of a sliding mode observer is simple and easy to implement. Because of the excellent robustness of the sliding mode variable techniques, the dependence on the precise mathematical model can be effectively reduced. The performance of the observer can be ensured in the case of a system modeling error, parameter perturbation, and the unknown inputs such as external noise and disturbance. Therefore, it has very strong engineering practicability.

Consider a sliding mode surface:

$$
s=F e_{y}(t)
$$

Proposition 4 implies that the sliding mode dynamics of the error system (12) associated with the sliding surface (18) is stable. According to the sliding mode theory, observer stability will be guaranteed upon proving that the error system can be driven to the sliding mode surface in finite time by choosing an appropriate gain of $\rho$ for the input signals (11). In view of this, the conclusion is presented by the following proposition.

Proposition 6. If Assumptions 1-3 hold and $\rho$ is sufficiently large, then the error system (12) will be driven to the sliding mode surface in finite time.

Proof. Selecting the Lyapunov function

$$
V_{2}=\frac{1}{2} s^{T} s
$$

then the time derivative of $V_{2}$ along the trajectories of system (12) is

$$
\begin{aligned}
\dot{V}_{2} & =s^{T}(F \bar{C} \dot{e})=s^{T} F \bar{C}\left(A_{0} e+M\left(v-f_{s}\right)\right) \\
& =s^{T} F \bar{C} A_{0} e+s^{T} F \bar{C} M\left(v-f_{s}\right) \\
& =s^{T} F \bar{C} A_{0} e+s^{T} M^{T} P M\left(v-f_{s}\right) \\
& =s^{T} F \bar{C} A_{0} e-s^{T} M^{T} P M f_{s}+s^{T} M^{T} P M v \\
& =s^{T} F \bar{C} A_{0} e-s^{T} M^{T} P M f_{s}-\rho s^{T} M^{T} P M \frac{s}{\|s\|} .
\end{aligned}
$$

Thus, from (17), there is

$$
\begin{aligned}
\dot{V}_{2} & \leq\|s\|\left\|F \bar{C} A_{0}\right\|\|e\|+\|s\|\left\|M^{T} P M\right\|\left\|f_{s}\right\| \\
& -\rho \lambda_{\min }\left(M^{T} P M\right)\|s\| \leq\|s\|\left(\left\|F \bar{C} A_{0}\right\| \delta_{1}\right. \\
& \left.+\left\|M^{T} P M\right\|\left\|f_{s}\right\|-\rho \lambda_{\min }\left(M^{T} P M\right)\right) \leq-\|s\| \\
& \cdot \lambda_{\min }\left(M^{T} P M\right)(\rho \\
& \left.-\left(\frac{\left\|M^{T} P M\right\|}{\lambda_{\min }\left(M^{T} P M\right)}\left\|f_{s}\right\|+\frac{\left\|F \bar{C} A_{0}\right\|}{\lambda_{\min }\left(M^{T} P M\right)} \delta_{1}\right)\right) .
\end{aligned}
$$

The variables can be defined as

$$
\begin{aligned}
K & =\lambda_{\min }\left(M^{T} P M\right)(\rho \\
& \left.-\left(\frac{\left\|M^{T} P M\right\|}{\lambda_{\min }\left(M^{T} P M\right)}\left\|f_{s}\right\|+\frac{\left\|F \bar{C} A_{0}\right\|}{\lambda_{\min }\left(M^{T} P M\right)} \delta_{1}\right)\right) .
\end{aligned}
$$

Since $\left\|M^{T} P M\right\| \geq \lambda_{\min }\left(M^{T} P M\right)>0$, it follows that $\left(\left\|M^{T} P M\right\| / \lambda_{\min }\left(M^{T} P M\right)\right)\left\|f_{s}\right\|+\left(\left\|F \bar{C} A_{0}\right\| / \lambda_{\min }\left(M^{T} P M\right)\right) \delta_{1} \geq$ $\left\|f_{s}\right\|$. So the scalar $\rho$ in (11) satisfies $\rho>\rho_{1}$, and $\rho$ can be selected to be large enough to satisfy

$$
\rho \geq \frac{\left\|M^{T} P M\right\|}{\lambda_{\min }\left(M^{T} P M\right)} \rho_{1}+\frac{\left\|F \bar{C} A_{0}\right\|}{\lambda_{\min }\left(M^{T} P M\right)} \delta_{1} ;
$$

then

$$
\dot{V}_{2} \leq-K\|s\| \text {. }
$$

This shows that the sliding mode reachability condition is satisfied. As a consequence, according to sliding mode principle [15], an ideal sliding motion will take place on the surface $s$ after some finite time.

This completes the proof.

When the system reaches the sliding mode surface, $s=$ $\dot{s}=0$ according to the sliding mode equivalent principle [9]. This implies

$$
\begin{aligned}
F \bar{C} e(t) & =F e_{y}(t)=0, \\
F \bar{C} \dot{e} & =F \dot{e}_{y}(t)=F e_{y}(t)=0
\end{aligned}
$$


and, from (12) and (25), the fault reconstruction equation can be got in the form of

$$
f_{s}=v
$$

that is, the fault is now equivalent to the sliding mode signal.

Remark 7. Unlike many other methods which use residuals to diagnose the occurrence of sensor fault qualitatively, the method of fault reconstruction presented in this paper estimates the current sensor fault quantitatively. By this way, not only the original appearance of a fault can be reflected vividly, but also more specific fault information can be obtained. It actually becomes the important basis of adopting more targeted measures to eliminate the effect of fault on PMSM drives or achieving the active fault-tolerant control proposal as presented in this paper.

3.2. Reconstructing the Phase Current Sensors Fault Signals. As mentioned previously, a fault on one of the $\alpha-\beta$ axis current sensors can be reconstructed. But this design will not provide more specific information whether the faulty sensor is in phase " $a$ " or " $b$." Therefore, the phase current sensor fault isolation and identification become somewhat complicated. To overcome this problem, a logic algorithm is constructed, devoted to transforming the $\alpha-\beta$ axis fault signals in $a b c$ phase fault signals of PMSM. Thus, the phase current sensor faults will be reconstructed.

Generally, for electric motors, only two phase sensors are used. That is based on considerations of cost and the fact that the three phase currents make a vector sum to zero in a star connected system without a neutral line. So, in actual control systems of PMSM, the two phase stator currents, $i_{a}$ and $i_{b}$, are measured by the phase current sensors, respectively, and the third phase current, $i_{c}$, is calculated using $i_{a}+i_{b}+i_{c}=0$.

A linear (3/2) Clarke transformation is applied to transform the three-phase plane coordinate system $A B C$ to the two-phase plane rectangular coordinate system $\alpha \beta$, the transformation equation is as below:

$$
\left[\begin{array}{l}
i_{\alpha} \\
i_{\beta} \\
i_{0}
\end{array}\right]=\sqrt{\frac{2}{3}}\left[\begin{array}{ccc}
1 & -\frac{1}{2} & -\frac{1}{2} \\
0 & \frac{\sqrt{3}}{2} & -\frac{\sqrt{3}}{2} \\
\frac{1}{\sqrt{2}} & \frac{1}{\sqrt{2}} & \frac{1}{\sqrt{2}}
\end{array}\right]\left[\begin{array}{l}
i_{a} \\
i_{b} \\
i_{c}
\end{array}\right]
$$

where $i_{0}$ is a variable introduced, distinct from $i_{a}$ and $i_{b}$, used for construction of the $\alpha-\beta-0$ coordinate system, called the zero-axis current. The zero-axis is vertical to both $\alpha$ and $\beta$ axes, and so it will have no influence on $\alpha$ and $\beta$ axes.

For star connected systems without a neutral line, $i_{a}+i_{b}+$ $i_{c}=0$; that is, $i_{c}=-\left(i_{a}+i_{b}\right)$. Equation (27) becomes

$$
\left[\begin{array}{l}
i_{\alpha} \\
i_{\beta}
\end{array}\right]=\left[\begin{array}{cc}
\sqrt{\frac{3}{2}} & 0 \\
\frac{\sqrt{2}}{2} & \sqrt{2}
\end{array}\right]\left[\begin{array}{l}
i_{a} \\
i_{b}
\end{array}\right] .
$$

It can be seen that decoupling can be realized for current in this equation.

As discussed above, practically, currents $i_{\alpha}$ and $i_{\beta}$ are calculated from phase currents $i_{a}$ and $i_{b}$. Then, from (28), the effects of the stator $\alpha-\beta$ axis currents are related to the errors in phase $a$ and $b$ current sensors outputs:

$$
\left[\begin{array}{c}
i_{\alpha}+f_{s \alpha} \\
i_{\beta}+f_{s \beta}
\end{array}\right]=\left[\begin{array}{cc}
\sqrt{\frac{3}{2}} & 0 \\
\frac{\sqrt{2}}{2} & \sqrt{2}
\end{array}\right]\left[\begin{array}{l}
i_{a}+f_{s a} \\
i_{b}+f_{s b}
\end{array}\right],
$$

where $f_{s \alpha}$ and $f_{s \beta}$ are the $\alpha$ - $\beta$ axis sensor faults, respectively, and $f_{s a}$ and $f_{s b}$ are the $a$ and $b$ phase sensor faults, respectively.

From (28) and (29), the phase sensor faults $f_{s a}$ and $f_{s b}$ are

$$
\begin{aligned}
& f_{s a}=\sqrt{\frac{2}{3}} f_{s \alpha}, \\
& f_{s b}=\frac{1}{\sqrt{2}} f_{s \beta}-\sqrt{\frac{1}{6}} f_{s \alpha},
\end{aligned}
$$

where $f_{s \alpha}, f_{s \beta}$ are derived by reconstruction of the state observer in the previous section.

In addition, the actual phase current is

$$
\begin{aligned}
& i_{a}=\sqrt{\frac{2}{3}} i_{\alpha}, \\
& i_{b}=\frac{1}{\sqrt{2}} i_{\beta}-\sqrt{\frac{1}{6}} i_{\alpha} .
\end{aligned}
$$

Thus, the phase current sensor fault signals can be reconstructed by transformation of the $\alpha$ - $\beta$ axis fault signals of a PMSM.

Remark 8. In the previous literature $[5,6]$, the current sensor fault detection was usually corresponding to $d$ - $q$ axis, which is a virtual axis. In contrast, this paper proposes a new current sensor FDI algorithm which is directly corresponding to $a b c$-phase, the actual mounting position of current sensor. This innovation can significantly improve the practicability of traditional FDI algorithm, as well as bring many potential applications.

\section{Simulation Study}

To verify the effectiveness of the method proposed in this paper, the drive tests with respect to two types of faults have been carried out. One tested fault is the incipient fault and the other is the gain sensor fault. The parameters for the PMSM of this study are given in Table 1 .

The matrixes $A_{s}$ and $B_{s}$ in (4) are then

$$
\begin{aligned}
& A_{s}=\left[\begin{array}{cc}
-200 & 0 \\
0 & -200
\end{array}\right], \\
& B_{s}=\left[\begin{array}{cc}
200 & 0 \\
0 & 200
\end{array}\right] .
\end{aligned}
$$




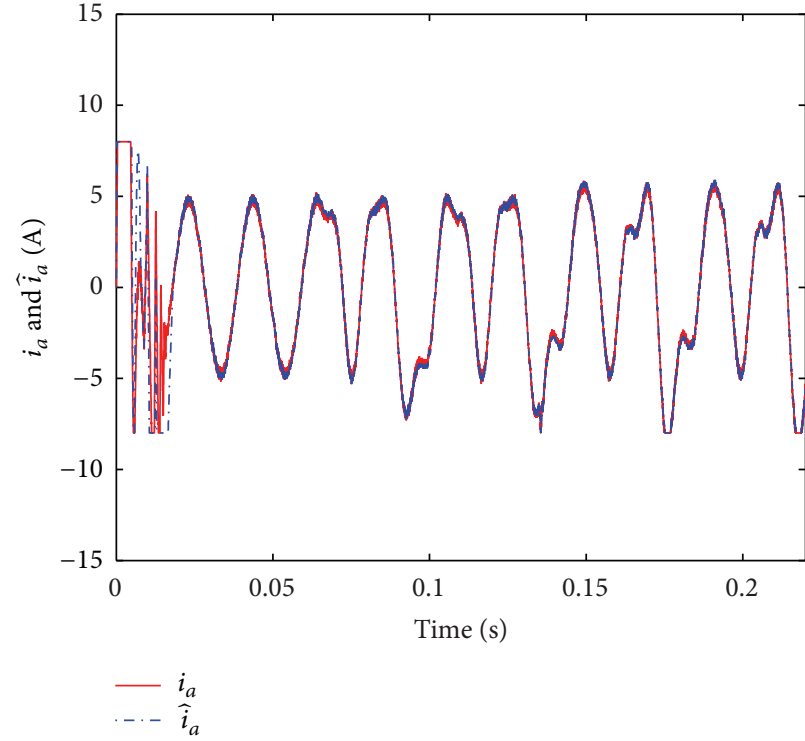

(a) $i_{a}$ and its estimation $\hat{i}_{a}$

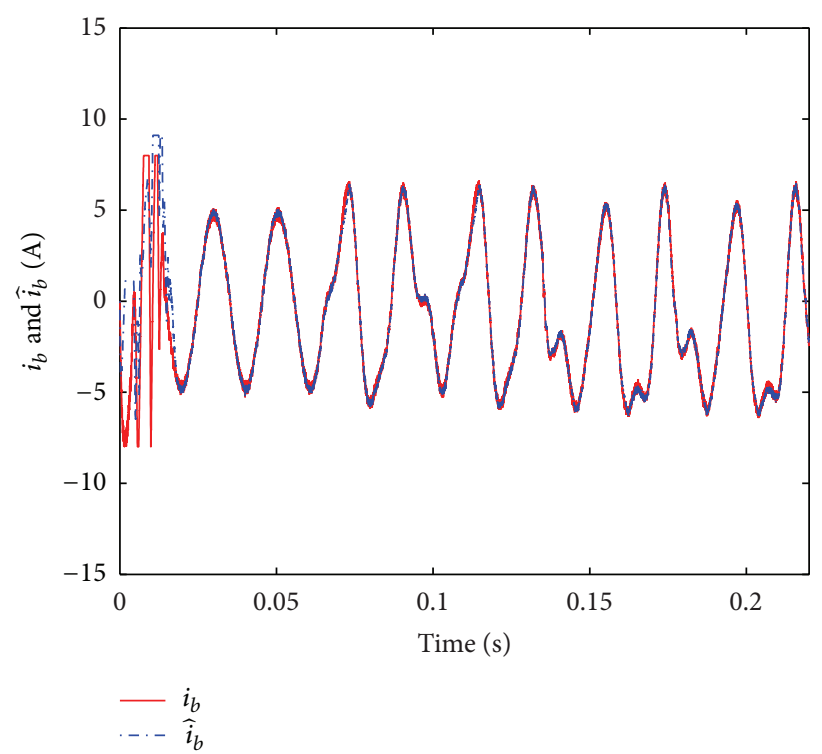

(b) $i_{b}$ and its estimation $\hat{i}_{b}$

FIgURE 1: Case 1: current estimation.

TABLE 1: Nominal value for PMSM.

\begin{tabular}{lcc}
\hline Parameters & Unit & Values \\
\hline Stator resistance $\left(R_{s}\right)$ & $\Omega$ & 2.875 \\
Number of pole pairs $\left(n_{p}\right)$ & Pairs & 4 \\
Stator inductance $\left(L_{s}\right)$ & $\mathrm{H}$ & 0.0085 \\
Rotor PM flux $\left(\psi_{f}\right)$ & $\mathrm{Wb}$ & 0.175 \\
Rotor moment of inertia $(J)$ & $\mathrm{kg} \cdot \mathrm{m}^{2}$ & 0.008 \\
\hline
\end{tabular}

The matrixes in system (8) are

$$
\begin{aligned}
& \bar{A}=\left[\begin{array}{cccc}
-338.23 & 0 & 0 & 0 \\
0 & -338.23 & 0 & 0 \\
200 & 0 & -200 & 0 \\
0 & 200 & 0 & -200
\end{array}\right], \\
& \bar{B}=\left[\begin{array}{cc}
117.65 & 0 \\
0 & 117.65 \\
0 & 0 \\
0 & 0
\end{array}\right] \text {, } \\
& \bar{C}=\left[\begin{array}{llll}
0 & 0 & 1 & 0 \\
0 & 0 & 0 & 1
\end{array}\right], \\
& \bar{E}=\left[\begin{array}{cc}
-117.65 \omega & 0 \\
0 & -117.65 \omega \\
0 & 0 \\
0 & 0
\end{array}\right] \text {, }
\end{aligned}
$$

$$
M=\left[\begin{array}{cc}
0 & 0 \\
0 & 0 \\
200 & 0 \\
0 & 200
\end{array}\right]
$$

and matrix $(\bar{A}, \bar{C})$ is observable since $B_{s}$ is nonsingular.

The matrix $L$ in (10) is

$$
L=\left[\begin{array}{cccc}
100 & 0 & 200 & 0 \\
0 & 100 & 0 & 100
\end{array}\right]^{T}
$$

And so the corresponding $A_{0}$ matrix is

$$
A_{0}=\left[\begin{array}{cccc}
-338.23 & 0 & -100 & 0 \\
0 & -338,23 & 0 & -100 \\
200 & 0 & -400 & 0 \\
0 & 200 & 0 & -100
\end{array}\right]
$$

and, using linear matrix inequality techniques, we have the following solution:

$$
\begin{aligned}
& P=\left[\begin{array}{cccc}
724.4 & 0 & 0 & 0 \\
0 & 759.5 & 0 & 0 \\
0 & 0 & 567.4 & 0 \\
0 & 0 & 0 & 745.5
\end{array}\right], \\
& F=\left[\begin{array}{ccc}
113480 & 0 \\
0 & 149100
\end{array}\right] .
\end{aligned}
$$






(a) The fault $f_{s a}$ and its estimation

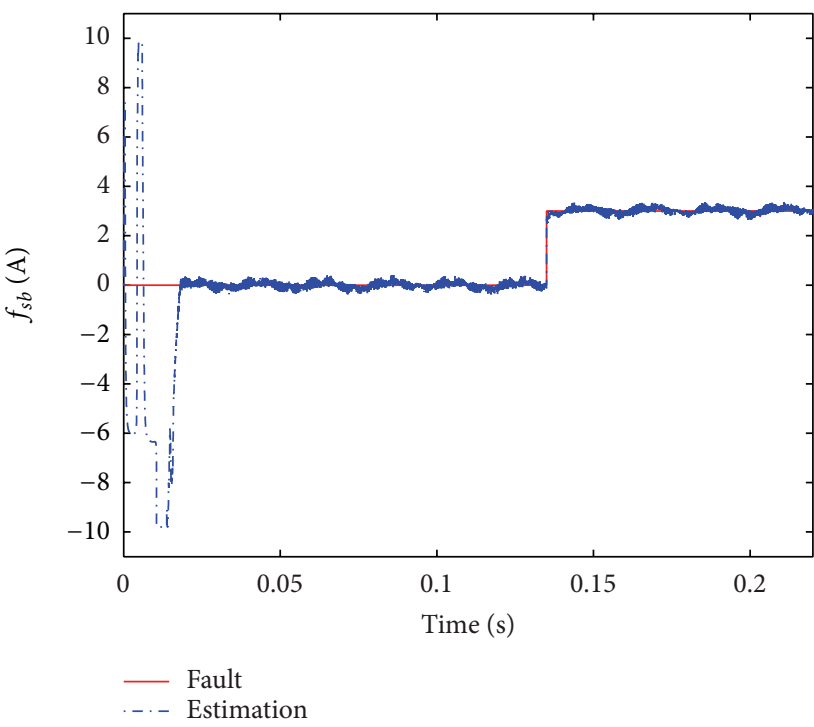

(b) The fault $f_{s b}$ and its estimation

FIGURE 2: Case 1: current sensor faults estimation.

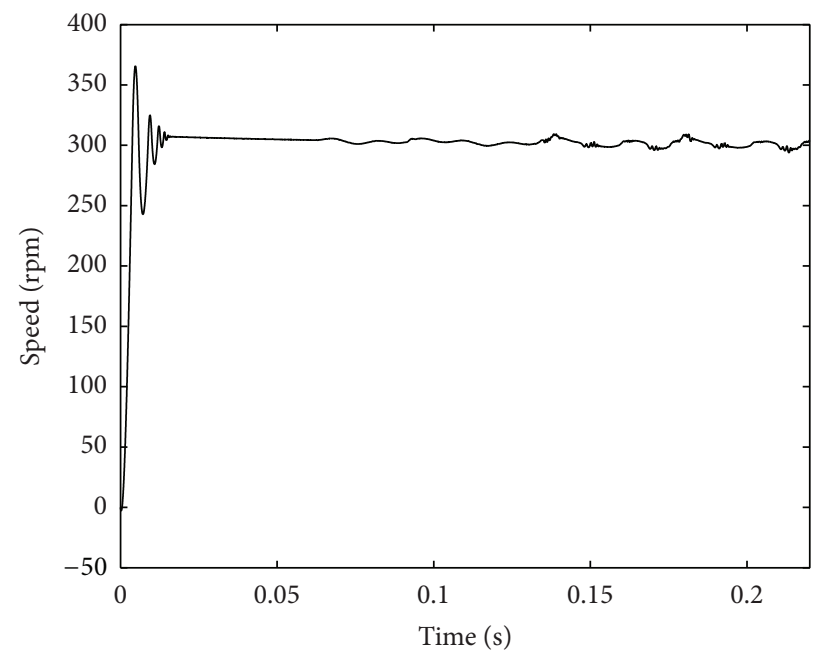

FIgURE 3: Case 1: the motor speed waveform during current sensor faults on $a$ and $b$ phases.

Remark 9. The following saturation function can be used to substitute for the sliding mode signal $v$ to reduce chattering and eliminate high-frequency interference caused by the chattering:

$$
v=-\rho \frac{F e_{y}}{\left\|F e_{y}\right\|+\delta_{2}},
$$

where $\delta_{2}$ is a scalar of relatively small value.

The following parameters are chosen: $\rho=46, \delta_{2}=0.1$; initial simulation conditions are all $5 \mathrm{~A}$; output limiting values of state variable are chosen as $\pm 10 \mathrm{~A}$; fault reconstruction limiting values are chosen as $\pm 7 \mathrm{~A}$; simulation time is $0.22 \mathrm{~s}$; and the given speed is $300 \mathrm{rpm}$.
Case 1. A sinusoidal signal is added with amplitude $5 \mathrm{~A}$ and frequency $150 \mathrm{~Hz}$ for $a$-phase from $0.063 \mathrm{~s}$, simulating an incipient fault; and a step signal with amplitude $3 \mathrm{~A}$ from $0.135 \mathrm{~s}$ for $b$-phase, simulating a gain sensor fault.

The simulation (Figures 1-2) shows that the fault signals can be accurately reconstructed. The faults produce motor speed abnormalities in the drive's operation, as shown in Figure 3.

Case 2. A step signal is added with amplitude $5 \mathrm{~A}$ from $0.063 \mathrm{~s}$ for $a$-phase, simulating a gain sensor fault; and a sinusoidal signal with amplitude $3 \mathrm{~A}$ and frequency $150 \mathrm{~Hz}$ for $b$-phase from $0.135 \mathrm{~s}$, simulating an incipient fault.

The simulation (Figures 4-5) shows that the fault signals can be accurately reconstructed. The faults produced motor 


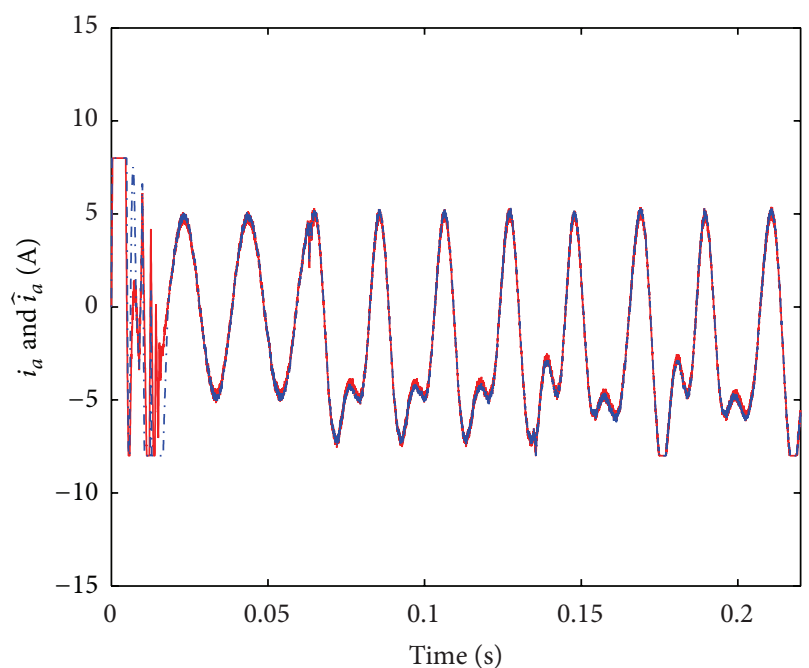

$-i_{a}$

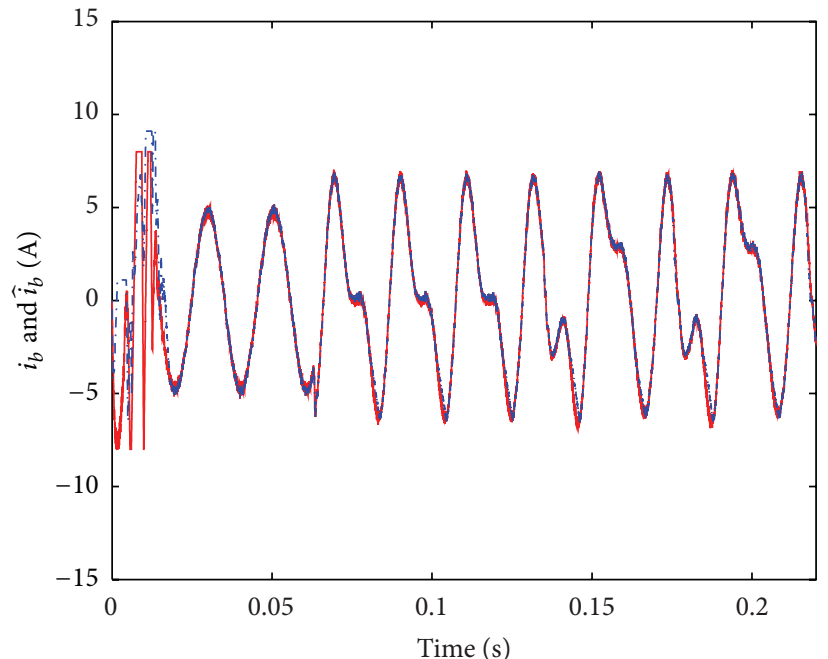

$-i_{b}$

(a) $i_{a}$ and its estimation $\hat{i}_{a}$

(b) $i_{b}$ and its estimation $\hat{i}_{b}$

FIgURE 4: Case 2: current estimation.

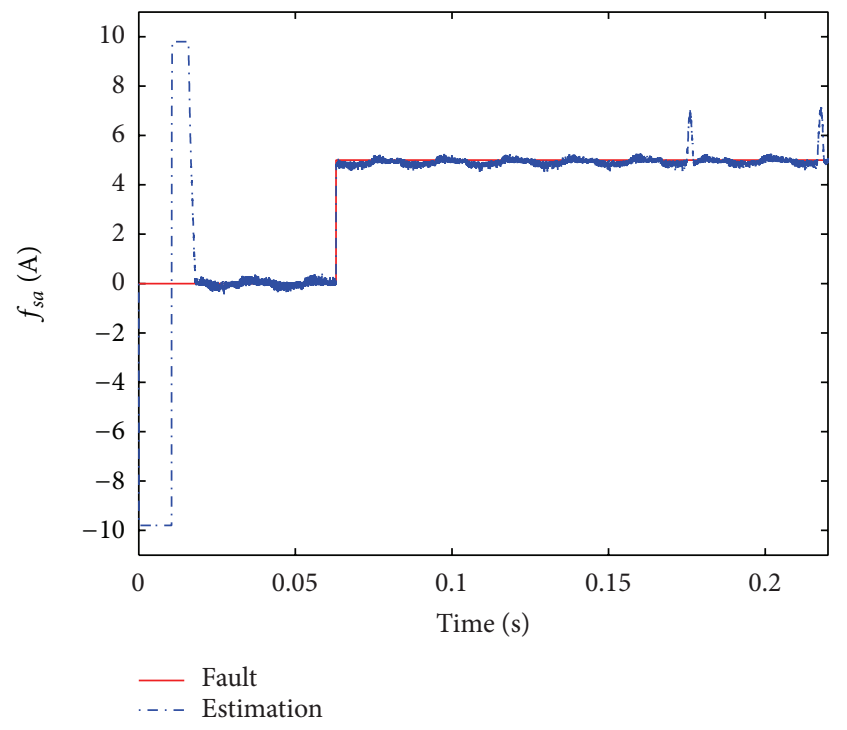

(a) The fault $f_{s a}$ and its estimation

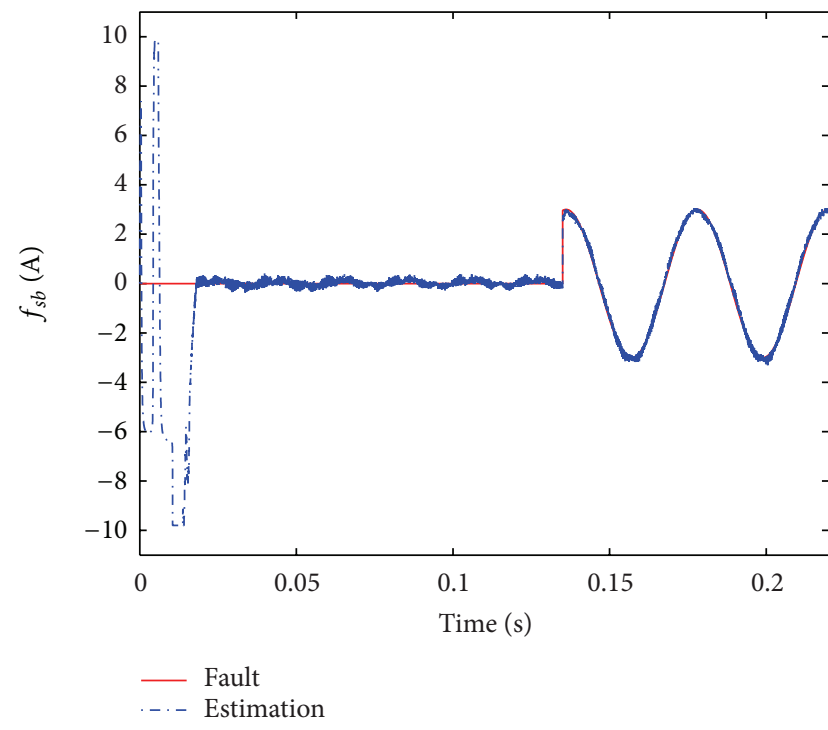

(b) The fault $f_{s b}$ and its estimation

FIGURE 5: Case 2: current sensor faults estimation.

speed abnormalities in the drive's operation, as shown in Figure 6.

The simulations show that the fault reconstruction is realized. The fault signal can be estimated to determine the size, location, and time of occurrence of a fault on phase $a$ or $b$ current sensor of a PMSM intuitively. This method will be ideal for directly isolating the faulty current sensor.

\section{Conclusions}

This paper presents a PMSM phase current sensor fault reconstruction method based on sliding mode variable structure observer. An $\alpha$ - $\beta$ axis fault model of PMSM is firstly defined. Based on this, a first-order low-pass filter is introduced for constructing an augmented system with which the PMSM sensor faults can be considered as actuator faults. 


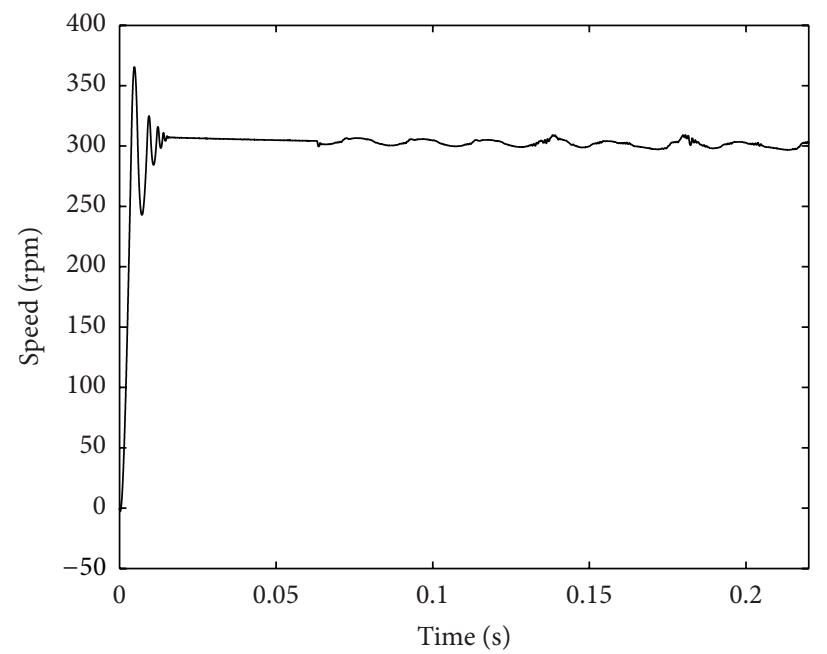

FIgURE 6: Case 2: the motor speed waveform during current sensor faults on $a$ and $b$ phases.

The design of a sliding mode variable structure observer follows to achieve fault reconstruction by using sliding mode equivalent principle. Then it comes to the design of logic algorithm, with which the reconstructed $\alpha$ - $\beta$ axis fault signal can be converted into $a b c$-phase, and then the detection and reconstruction of actual fault of phase current sensor can be implemented. It can be seen from the proof and simulation results that, with the proposed fault reconstruction scheme, there is almost no restriction on the fault type. In other words, the scheme is applicable for abrupt fault, incipient fault, or any other types of faults.

\section{Conflict of Interests}

The authors declare that there is no conflict of interests regarding the publication of this paper.

\section{Acknowledgments}

This work was supported by the Natural Science Foundation of China (nos. 61273157 and 61473117), Hunan Provincial Natural Science Foundation of China (nos. 14JJ5024 and 2015JJ5011), and Hunan Province Education Department Project of China (nos. 12A040 and 13CY018).

\section{References}

[1] I. Aydin, M. Karakose, and E. Akin, "An approach for automated fault diagnosis based on a fuzzy decision tree and boundary analysis of a reconstructed phase space," ISA Transactions, vol. 53, no. 2, pp. 220-229, 2014.

[2] H. Lu, X. Cheng, W. Qu, S. Sheng, Y. Li, and Z. Wang, "A three-phase current reconstruction technique using single DC current sensor based on TSPWM," IEEE Transactions on Power Electronics, vol. 29, no. 3, pp. 1542-1550, 2014.

[3] F. R. Salmasi and T. A. Najafabadi, "An adaptive observer with online rotor and stator resistance estimation for induction motors with one phase current sensor," IEEE Transactions on Energy Conversion, vol. 26, no. 3, pp. 959-966, 2011.
[4] T. A. Najafabadi, F. R. Salmasi, and P. Jabehdar-Maralani, "Detection and isolation of speed-, DC-link voltage-, and currentsensor faults based on an adaptive observer in induction-motor drives," IEEE Transactions on Industrial Electronics, vol. 58, no. 5, pp. 1662-1672, 2011.

[5] L. Liu, Robust fault detection and diagnosis for permanent magnet synchronous motors [A Thesis Presented for the Degree of Doctor of Philosophy], The Florida State University, Summer Semester, 2006.

[6] Y. Huang, Fault diagnosis method based on observer and application research [M.S. thesis], The Hunan University of Technology, Summer Semester, 2011.

[7] A. Šabanovic, "Variable structure systems with sliding modes in motion control-a survey," IEEE Transactions on Industrial Informatics, vol. 7, no. 2, pp. 212-223, 2011.

[8] Z. Gao, B. Jiang, P. Shi, M. Qian, and J. Lin, "Active fault tolerant control design for reusable launch vehicle using adaptive sliding mode technique," Journal of the Franklin Institute, vol. 349, no. 4, pp. 1543-1560, 2012.

[9] B. Jiang, M. Staroswiecki, and V. Cocquempot, "Fault estimation in nonlinear uncertain systems using robust/sliding-mode observers," IEE Proceedings: Control Theory and Applications, vol. 151, no. 1, pp. 29-37, 2004.

[10] C. Edwards, S. K. Spurgeon, and R. J. Patton, "Sliding mode observers for fault detection and isolation," Automatica, vol. 36, no. 4, pp. 541-553, 2000.

[11] C. P. Tan and C. Edwards, "Sliding mode observers for detection and reconstruction of sensor faults," Automatica, vol. 38, no. 10, pp. 1815-1821, 2002.

[12] H. K. Khalil, Nonlinear Systems, Prentice-Hall, Englewood Cliffs, NJ, USA, 3rd edition, 2002.

[13] X.-G. Yan and C. Edwards, "Sensor fault detection and isolation for nonlinear systems based on a sliding mode observer," International Journal of Adaptive Control and Signal Processing, vol. 21, no. 8-9, pp. 657-673, 2007.

[14] J. He, J. Qiu, and C. Zhang, "A multiple fault diagnosis method based on sliding mode observer," Journal of Dynamics and Control, vol. 7, no. 1, pp. 84-91, 2009.

[15] F. Y. Wang, Sliding Mode Variable Structure Control, Tsinghua University Press, Beijing, China, 2005. 


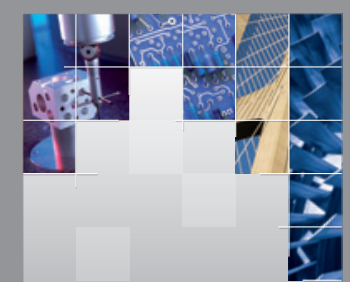

\section{Enfincering}
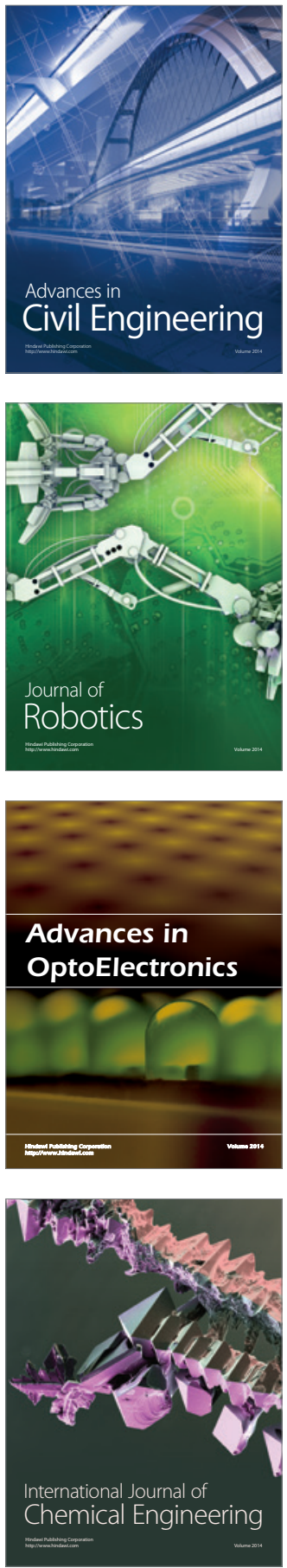

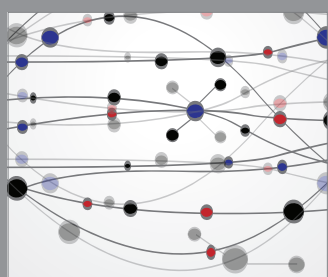

The Scientific World Journal

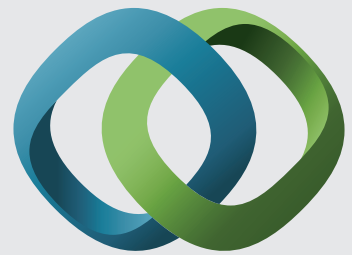

\section{Hindawi}

Submit your manuscripts at

http://www.hindawi.com
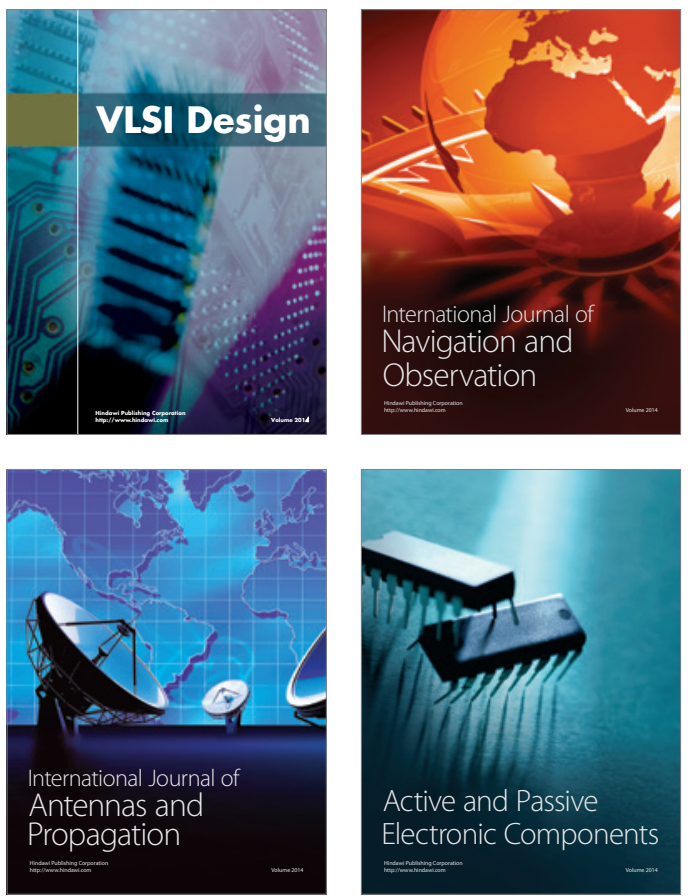
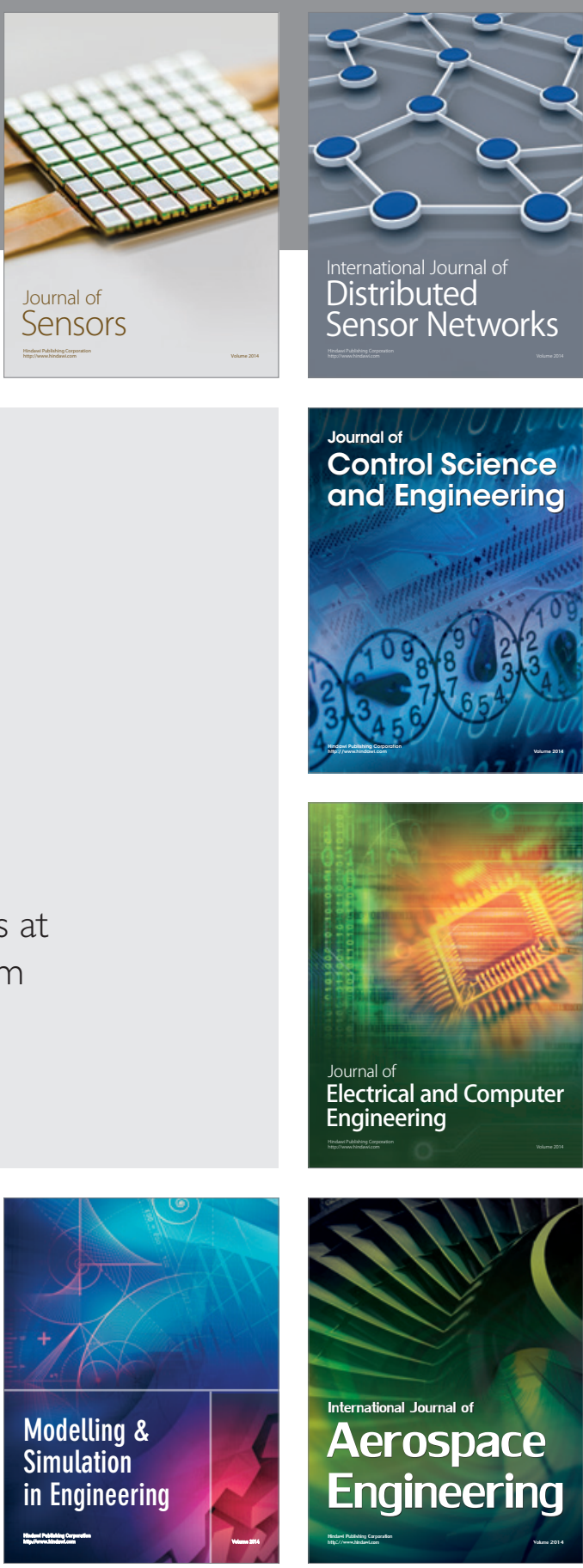

International Journal of

Distributed

Sensor Networks

Journal of

Control Science

and Engineering
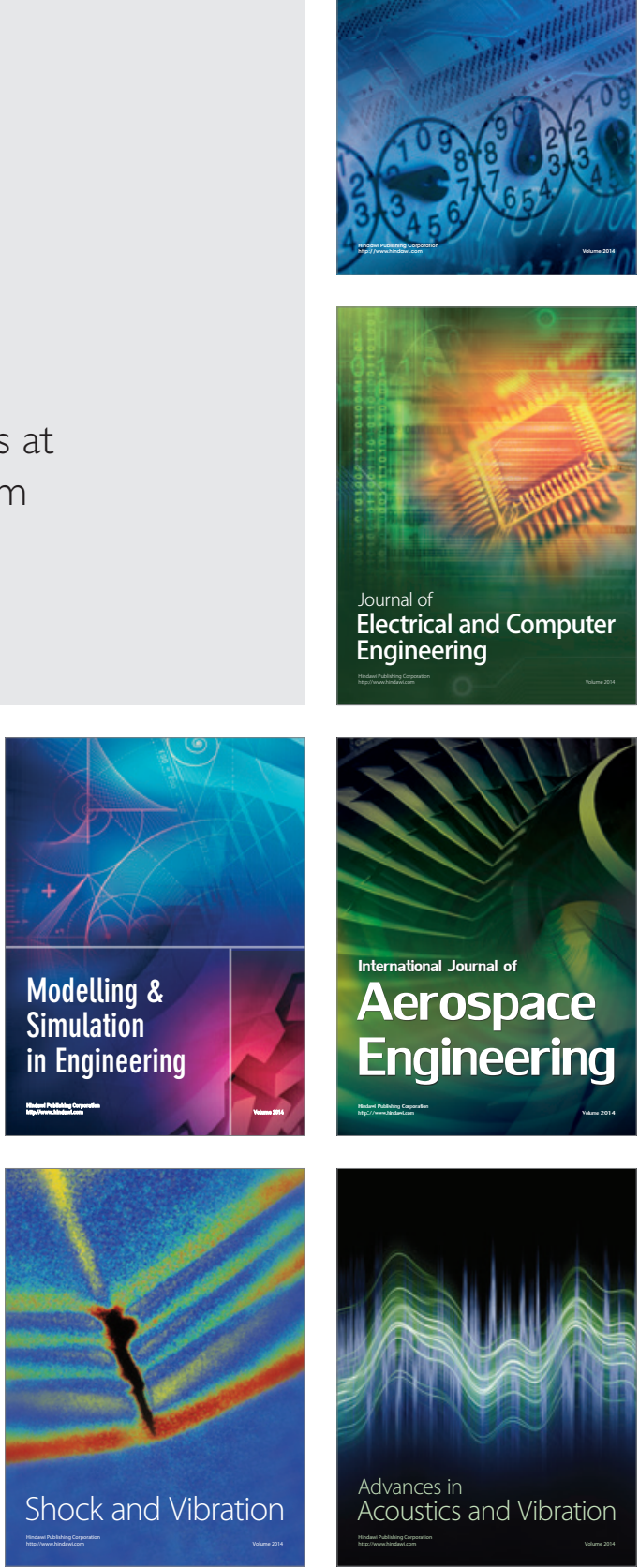